\title{
Paediatric hematopathology: something real special
}

\section{J. H. van Krieken ${ }^{1}$}

Published online: 25 July 2015

(C) Springer-Verlag Berlin Heidelberg 2015

The present issue of the Journal of Hematopathology deals with paediatric hematopathology. Some colleagues may wonder why this topic needs a special issue. Aren't haematological neoplasms in children not exceedingly rare and not too different from adults? The first answer is yes, the second no. Indeed, haematological neoplasms in children are very rare, but that supports the need of a special issue: few of us have a lot of experience and yet many hematopathologists occasionally encounter situations in which their call is very critical. In fact, the evaluation of haematological proliferations in children needs specific knowledge to prevent harm by unnecessary treatment on the one hand and install needed treatment quickly on the other. And indeed, the proliferations in children are different from adults. Overdiagnosis of a clonal population in a child has serious consequences and a (too) late diagnosis of an aggressive lymphoproliferation as well. The present special issue updates the reader on several recent and relevant developments.

One important question remains: why are lymphoproliferations in children so different from those that arise in adults? There is no general answer, except from the category of lymphoproliferations arising in congenital immune deficiencies. These are uncommon but also sometimes not recognised. They will be part of the SH/EAHP workshop later this year in Long Beach, California. Recognising them is a very rewarding task of any hematopathologist who encounters an unusual lesion in a child.

However, for most paediatric haematological neoplasms, we do not know why they occur in children and behave differently compared to similar lesions in adults. Even for Burkitt lymphoma and ALK-positive anaplastic large cell lymphoma, lesions of which we know very well the prime molecular background, we do not really understand why they occur typically in a child. Although these lesions are now well treatable, knowing the real aetiology would be quite helpful and maybe even make prevention an option. With all the new information that we collect nowadays, it remains a sobering thought that we still have very little real understanding why a certain individual develops a certain malignancy: much more research remains needed! Nevertheless, this special issue will help you making an optimal diagnosis, based on which a child will have the best chance.

Han van Krieken
J. H. van Krieken

Han.vanKrieken@radboudumc.nl

1 Department of Pathology, Radboud University Medical Centre, P.O. Box 9101, 6500, HB Nijmegen, The Netherlands 